\title{
Local Causality, Probability and Explanation
}

\author{
Richard A. Healey \\ The University of Arizona
}

January 8, 2016

\begin{abstract}
In papers published in the 25 years following his famous 1964 proof John Bell refined and reformulated his views on locality and causality. Although his formulations of local causality were in terms of probability, he had little to say about that notion. But assumptions about probability are implicit in his arguments and conclusions. Probability does not conform to these assumptions when quantum mechanics is applied to account for the particular correlations Bell argues are locally inexplicable. This account involves no superluminal action and there is even a sense in which it is local, but it is in tension with the requirement that the direct causes and effects of events are nearby.
\end{abstract}

\section{Introduction}

I never met John Bell, but his writings have supplied me with a continual source of new insights as I read and reread them over 40 years. In working toward a rather different understanding of quantum mechanics he has been foremost in my mind as a severe but honest critic of such attempts. We all would love to know what Einstein would have made of Bell's theorem. I confess the deep regret I feel that Bell cannot respond to this paper is sometimes assuaged by a sense of relief.

\section{Locality and Local Causality}

In his seminal 1964 paper, John Bell expressed locality as the requirement

that the result of a measurement on one system be unaffected by operations on a distant system with which it has interacted in the past. [2004, p. 14]

This seems to require that the result of a measurement would have been the same, no matter what operations had been performed on such a distant system. But suppose the result of a measurement were the outcome of an indeterministic process. Then the result of the measurement might have been different even 
if exactly the same operations (if any) had been performed on that distant system. So can no indeterministic theory satisfy the locality requirement? Bell felt no need to address that awkward question in his 1964 paper, since he took the EPR argument to establish that any additional variables needed to restore locality and causality would have to determine a unique result of a measurement. Indeterminism was not an option:

Since we can predict in advance the result of measuring any chosen component of $\boldsymbol{\sigma}_{2}$ [in the Bohm-EPR scenario], by previously measuring the same component of $\boldsymbol{\sigma}_{1}$, it follows that the result of any such measurement must actually be predetermined. (op. cit., p. 15)

Afterwards he repeatedly stressed that any theory proposed as an attempt to complete quantum theory while restoring locality and causality need not be assumed to be deterministic: to recover such perfect (anti)correlations it would have to be deterministic. This argument warrants closer examination, and I'll come back to it. But in later work Bell offered formulations of locality conditions tailored to theories that were not deterministic.

An initial motivation may have been to facilitate experimental tests of attempted local, causal completions of quantum mechanics by suitable measurements of spin or polarization components on pairs of separated systems represented by entangled quantum states. Inevitable apparatus imperfections would make it impossible to confirm a quantum prediction of perfect (anti)correlation for matched components, so experiment alone could not require a local, causal theory to reproduce them. Einstein himself thought some theory might come to underlie quantum mechanics much as statistical mechanics underlies thermodynamics. In each case there would be circumstances in which the more basic theory (correctly) predicts deviations from behavior the less basic theory leads one to expect.

But by 1975 a second motivation had become apparent - the hope that by revising or reformulating quantum mechanics as a theory of local beables one might remove ambiguity and arrive at increased precision. It is in this context that Bell now introduces a requirement of local causality. This differs in two ways from his earlier locality requirement. It is not a requirement on the world, but on theories of local beables: and it applies to theories that are probabilistic, with deterministic theories treated as a special case in which all probabilities are 0 or 1 (and densities are delta functions) 11 Bell ([1975], [1985]) designs his requirement of local causality as a generalization of a requirement of local determinism met by Maxwell's electromagnetic theory. In source-free Maxwellian electromagnetism, the local beables are the values of the electric and magnetic fields at each point $(\mathbf{x}, t)$. This theory is locally deterministic because

\footnotetext{
${ }^{1}$ While Bell did not make this explicit in 1975, his 1990 paper also notes the analogy with source-free Maxwellian electromagnetism, and there he does say:
}

The deterministic case is a limit of the probabilistic case, the probabilities becoming delta functions. [2004, p. 240] 
the field values in a space-time region are uniquely determined by their values at an earlier moment in a finite volume of space that fully closes the backward light cone of that region.

Local causality arises by generalizing to theories in which the assignment of values to some beables $\Lambda$ implies, not necessarily a particular value, but a probability distribution $\operatorname{Pr}(A \mid \Lambda)$, for another beable $A$. Here is how Bell ([2004, p. 54]) defines it (in my notation):

Let $N$ denote a specification of all the beables, of some theory, belonging to the overlap of the backward light cones of space-like separated regions 1 and 2 . Let $\Lambda$ be a specification of some beables from the remainder of the backward light cone of 1 , and $B$ of some beables in the region 2 . Then in a locally causal theory $\operatorname{Pr}(A \mid \Lambda, N, B)=\operatorname{Pr}(A \mid \Lambda, N)$ whenever both probabilities are given by the theory.

If $M$ is a specification of some beables from the backward light cone of 2 but not of 1 , then (assuming the joint probability distribution $\operatorname{Pr}(A, B \mid \Lambda, M, N)$ exists)

$$
\begin{aligned}
\operatorname{Pr}(A, B \mid \Lambda, M, N) & =\operatorname{Pr}(A \mid \Lambda, M, N, B) \cdot \operatorname{Pr}(B \mid \Lambda, M, N) \\
& =\operatorname{Pr}(A \mid \Lambda, N) \cdot \operatorname{Pr}(B \mid \Lambda, M)
\end{aligned}
$$

where (1) follows from the definition of conditional probability, and (2) follows for any locally causal theory. This means that any theory of local beables that is locally causal satisfies the condition

$$
\operatorname{Pr}(A, B \mid \Lambda, M, N)=\operatorname{Pr}(A \mid \Lambda, N) \cdot \operatorname{Pr}(B \mid \Lambda, M)
$$

In his 1990 presentation Bell modified his formulation of local causality, in part in response to constructive criticisms. He also defended his revised formulation by appeal to an Intuitive Principle of local causality (IP), namely

The direct causes (and effects) of events are near by, and even the indirect causes (and effects) are no further away than permitted by the velocity of light. [2004, p. 239]

Here is Bell's revised formulation of Local Causality (LC):

A theory will be said to be locally causal if the probabilities attached to values of local beables in a space-time region 1 are unaltered by specification of values of local beables in a space-like separated region 2, when what happens in the backward light cone of 1 is already sufficiently specified, for example by a full specification of local beables in a space-time region 3 [a thick "slice" that fully closes the backward light cone of region 1 wholly outside the backward light cone of 2]. (op. cit., p. 240). 
Bell [1990] then applies this condition to a schematic experimental scenario involving a linear polarization measurement on each photon in an entangled pair in which the polarizer setting $a$ and outcome recording $A$ for one photon occur in region 1 , while those $(b, B)$ for the other photon occur in region 2 . He derives a condition analogous to (3) and uses it to prove a CHSH inequality whose violation is predicted by quantum mechanics for the chosen entangled state for certain sets of choices of $a, b, 2$

$$
\operatorname{Pr}(A, B \mid a, b, c, \lambda)=\operatorname{Pr}(A \mid a, c, \lambda) \cdot \operatorname{Pr}(B \mid b, c, \lambda) \quad \text { (Factorizability) }
$$

Not only did this proof not assume the theory of local beables was deterministic, even this (Factorizability) condition was not assumed but derived from the reformulated local causality requirement.

Bell's formulation of local causality (LC) has been carefully analyzed by Norsen [2011], whose analysis has been further improved by Seevinck and Uffink [2011]. They have focused in their analyses on what exactly is involved in a sufficient specification of what happens in the backward light cone of 1 . This specification could fail to be sufficient through failing to mention local beables in 3 correlated with local beables in 2 through a joint correlation with local beables in the overlap of the backward light cones of 1 and 2. A violation of a local causality condition that did not require such a sufficient specification would pose no threat to the intuitive principle of local causality: specification of beables in 2 could alter the probabilities of beables in 1 if unspecified beables in 3 were correlated with both through a (factorizable) common cause in the overlap of the backward light cones of 1 and 2. On the other hand, requiring a specification of all local beables in 3, may render the condition (LC) inapplicable in attempting to show how theories meeting it predict correlations different from those successfully predicted by quantum theory.

To see the problem, consider the set-up for the intended application depicted in Figure 1. $A, B$ describe macroscopic event 3 , each usually referred to as the detection of a photon linearly polarized either vertically or horizontally relative to an $a$ - or $b$-axis respectively: $a, b$ label events at which an axis is selected by rotating through an angle $a^{\circ}, b^{\circ}$ respectively from some fixed direction in a plane. The region previously labeled 3 has been relabeled as $3 \mathrm{a}$, a matching region $3 \mathrm{~b}$ has been added in the backward light cone of 2 , and ' 3 ' now labels the entire continuous "stack" of space-like hypersurfaces right across the backward light cones of 1 and 2, shielding off these light cones' overlap from 1,2 themselves. Note that each of $1, a$ is space-like separated from each of $2, b$.

\footnotetext{
${ }^{2}$ Both $\lambda$ and $c$ are assumed confined to region 3 (now symmetrically extended so as also to close the backward light cone of 2): $c$ stands for the values of magnitudes characterizing the experimental set-up in terms admitted by ordinary quantum mechanics, while $\lambda$ specifies the values of magnitudes introduced by the theory supposed to complete quantum mechanics. It will not be necessary to mention $c$ in what follows.

${ }^{3}$ I use each of ' $A$ ', ' $B$ ' to denote a random variable with values $\left\{V_{A}, H_{A}\right\},\left\{V_{B}, H_{B}\right\}$ respectively. $e_{A}\left(\bar{e}_{A}\right)$ denotes the event in region 1 of Alice's photon being registered as vertically (horizontally) polarized. $e_{B}\left(\bar{e}_{B}\right)$ denotes the event in region 2 of Bob's photon being registered as vertically (horizontally) polarized.
} 
Figure 1:

In some theories, a complete specification of local beables in 3 would constrain (or even determine) the selection events $a, b$. But in the intended application $a, b$ must be treated as free variables in the following sense: in applying a theory to a scenario of the relevant kind each of $a, b$ is to be specifiable independently in a theoretical model, and both are taken to be specifiable independently of a specification of local beables in region 3. Since this may exclude some complete theoretical specifications of beables in region 3 it is best not to require such completeness. Instead, one should say exactly what it is for a specification to be sufficient.

Seevinck and Uffink [2011] clarify this notion of sufficiency as a combination of functional and statistical sufficiency, rendering the label $b$ and random variable $B$ (respectively) redundant for predicting $\operatorname{Pr}_{a, b}(A \mid B, \lambda)$, the probability a theory specifies for beable $A$ representing the outcome recorded in region 1 given beables $a, b$ representing the free choices of what the apparatus settings are in sub-regions of 1,2 respectively, conditional on outcome $B$ in region 2 and beable specification $\lambda$ in region 3 . This implies

$$
\operatorname{Pr}_{a, b}(A \mid B, \lambda)=\operatorname{Pr}_{a}(A \mid \lambda)
$$

Notice that $a, b$ are no longer treated as random variables, as befits their status as the locus of free choice. It would be unreasonable to require a theory of local beables to predict the probability that the experimenters make one free choice rather than another: but treating $a, b$ as random variables (as in Bell's formulation of Factorizability would imply the existence of probabilities of the

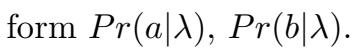


By symmetry, interchanging ' 1 ' with ' 2 ', ' $A$ ' with ' $B$ ' and ' $a$ ' with 'b' implies

$$
\operatorname{Pr}_{a, b}(B \mid A, \lambda)=\operatorname{Pr}_{b}(B \mid \lambda)
$$

Seevinck and Uffink [2011] offer equations (4a) and (4b) as their mathematically sharp and clean (re)formulation of the condition of local causality. Together, these equations imply the condition

$$
\operatorname{Pr}_{a, b}(A, B \mid \lambda)=\operatorname{Pr}_{a}(A \mid \lambda) \times \operatorname{Pr}_{b}(B \mid \lambda) \quad\left(\text { Factorizability }_{S U}\right)
$$

used to derive CHSH inequalities. Experimental evidence that these inequalities are violated by the observed correlations in just the way quantum theory leads one to expect may then be taken to disconfirm Bell's intuitive causality principle.

In more detail, Seevinck and Uffink [2011] claim that orthodox quantum mechanics violates the statistical sufficiency conditions (commonly known as Outcome Independence, following Shimony)

$$
\begin{aligned}
& \operatorname{Pr}_{a, b}(A \mid B, \lambda)=\operatorname{Pr}_{a, b}(A \mid \lambda) \\
& \operatorname{Pr}_{a, b}(B \mid A, \lambda)=\operatorname{Pr}_{a, b}(B \mid \lambda)
\end{aligned}
$$

while conforming to the functional sufficiency conditions (commonly known as Parameter Independence, following Shimony)

$$
\begin{aligned}
& \operatorname{Pr}_{a, b}(A \mid \lambda)=\operatorname{Pr}_{a}(A \mid \lambda) \\
& \operatorname{Pr}_{a, b}(B \mid \lambda)=\operatorname{Pr}_{b}(B \mid \lambda)
\end{aligned}
$$

Statistical sufficiency is a condition employed by statisticians in situations where considerations of locality and causality simply don't arise. But in this application the failure of quantum theory to provide a specification of beables in region 3 such that the outcome $B$ is always redundant for determining the probability of outcome $A$ (and similarly with ' $A$ ', ' $B$ ' interchanged) has clear connections to local causality, as Seevinck and Uffink's [2011] analysis has shown.

In the light of Seevinck and Uffink's [2011] analysis, perhaps Bell's local causality condition (LC) should be reformulated as follows:

$\left(\mathrm{LC}_{S U}\right) \quad$ A theory is said to be locally causal ${ }_{S U}$ if it acknowledges a class $R_{\lambda}$ of beables $\lambda$ in space-time region 3 whose values may be attached independently of the choice of $a, b$ and are then sufficient to render $b$ functionally redundant and $B$ statistically redundant for the task of specifying the probability of $A$ in region 1 .

The notions of statistical and functional redundancy appealed to here are as follows:

For $\lambda \varepsilon R_{\lambda}, \lambda$ renders $B$ statistically redundant for the task of specifying the probability of $A$ iff $\operatorname{Pr}_{a, b}(A \mid B, \lambda)=P r_{a, b}(A \mid \lambda)$.

For $\lambda \varepsilon R_{\lambda}, \lambda$ renders $b$ functionally redundant for the task of specifying the probability of $A$ iff $\operatorname{Pr}_{a, b}(A \mid \lambda)=\operatorname{Pr}_{a}(A \mid \lambda)$. 
Though admittedly less general than (LC), (LC) $S U$ seems less problematic but just as well motivated by (IP), as applied to the scenario depicted in Figure 1. If correlations in violation of the $\mathrm{CHSH}$ inequality are locally inexplicable in so far as no theory of local beables can explain them consistent with (LC), then they surely also count as locally inexplicable in so far as no theory of local beables can explain them consistent with $(\mathrm{LC})_{S U}$. But Bell himself said we should regard his step from (IP) to (LC) with the utmost suspicion, and that is what I shall do. My grounds for suspicion are my belief that quantum mechanics itself helps us to explain the particular correlations violating CHSH inequalities that Bell [2004, pp. 151-2] claimed to be locally inexplicable without action at a distance. Moreover, that explanation involves no superluminal action, and there is even a sense in which it is local.

To assess the status of (LC) (or $(\mathrm{LC})_{S U}$ ) in quantum mechanics one needs to say first how it is applied to yield probabilities attached to values of local beables in a space-time region 1 and then what it would be for these to be altered by specification of values of local beables in a space-like separated region 2. This is not a straightforward matter. The Born rule may be correctly applied to yield more than one chance for the same event in region 1, and there is more than one way to understand the requirement that these chances be unaltered by what happens in region 2. As we'll see, the upshot is that while Born rule probabilities do violate (Factorizability) (or (Factorizability $\left.{ }_{S U}\right)$ ) here, this counts as a violation of (LC) (or (LC) $S U$ ) only if that condition is applied in a way that is not motivated by (IP)'s prohibition of space-like causal influences.

\section{Probability and chance}

Bell credited his formulation of local causality (LC) with avoiding the "rather vague notions" of cause and effect by replacing them with a condition of probabilistic independence. The connection to (IP)'s motivating talk of 'cause' and 'effect' is provided by the thought that a cause alters (and typically raises) the chance of its effect. But this connection can be made only by using the general probabilities supplied by a theory to supply chances of particular events.

By chance I mean the definite, single-case probability of an individual event such as rain tomorrow in Tucson. As in this example, its chance depends on when the event occurs - afterwards, it is always 0 or 1: and it may vary up until that time as history unfolds. Chance is important because of its conceptual connections to belief and action. The chance of $e$ provides an agent's best guide to how strongly to believe that $e$ occurs, when not in a position to be certain that it does 4 And the comparison between $e$ 's chances according as (s)he does or does not do $D$ are critical in the agent's decision about whether to do $D$. These connections explain why the chance of an event defaults to 0 or 1 when the agent is in a position to be certain about it - typically, after it does or doesn't occur.

\footnotetext{
${ }^{4}$ I use the "tenseless present" rather than the more idiomatic future tense here for reasons that will soon become clear.
} 
Probabilistic theories may be useful guides to the chances of events, but what they directly yield are not chances but general probabilities of the form $\operatorname{Pr}_{C}(E)$ for an event of type $E$ relative to reference class $C$. To apply such a general probability to yield the chance of $e$, you need to specify the type $E$ of $e$ and also the reference class $C$. A probabilistic theory may offer alternative specifications when applied to determine the chance of $e$, in which case it becomes necessary to choose the appropriate specifications. Actuarial tables may be helpful when estimating the chance that you will live to be 100, but you differ in all kinds of ways from every individual whose death figures in those tables. What you want is the most complete available specification of your situation: this may include much irrelevant information, but it's not necessary to exclude this since it won't affect the chance anyway. In Minkowski space-time, the conceptual connection between chance $(\mathrm{Ch})$ and the degree of belief $(\mathrm{Cr})$ it prescribes is captured in this version of David Lewis's Principal Principle that implicitly defines chance.5

The chance of $e$ at $p$, conditional on any information $I_{p}$ about the contents of $p$ 's past light cone satisfies: $C r_{p}\left(e / I_{p}\right)={ }_{d f} C h_{p}(e)$.

Now consider an agent who accepts quantum theory and wishes to determine the chance of the event $e_{A}$ that the next photon detected by Alice registers as vertically polarized $\left(V_{A}\right)$. Assuming that the state $\left|\Phi^{+}\right\rangle=$ $\frac{1}{\sqrt{2}}(|H\rangle|H\rangle+|V\rangle|V\rangle)$ was prepared and the settings $a, b$ chosen long before, the agent is also in a position to be certain what these were. The agent is then in a position to use the Born rule to determine the chance of $e_{A}$. But that chance must be relativized not just to a time, but (relativistically) to a space-time point. So consider the following diagram:

As this shows, if the outcome in region 2 is of type $V_{B}$ then $C h_{p}\left(e_{A}\right)=$ $C h_{p^{\prime}}\left(e_{A}\right)=C h_{r}\left(e_{A}\right)=\frac{1}{2}$, but $C h_{q}\left(e_{A}\right)=\cos ^{2} \angle a b$. These are the chances that follow from application of the Born rule to state $\left|\Phi^{+}\right\rangle$, given settings $a, b$. In each case the event $e_{A}$ of the next photon detected in 1 's registering as vertically polarized has been specified as of type $V_{A}$, and the specification of the reference class at least includes the state and settings. Specifically,

$$
\begin{gathered}
C h_{p}\left(e_{A}\right)=\operatorname{Pr}_{a, b}^{\Phi^{+}}\left(V_{A}\right)=\left\|\hat{P}^{A}(V) \Phi^{+}\right\|^{2}=\frac{1}{2} . \\
C h_{q}\left(e_{A}\right)=\operatorname{Pr}_{a, b}^{\Phi^{+}}\left(V_{A} \mid V_{B}\right) \equiv \frac{\operatorname{Pr}_{a, b}^{\Phi^{+}}\left(V_{A}, V_{B}\right)}{\operatorname{Pr}_{b}^{\Phi^{+}}\left(V_{B}\right)}=\frac{\frac{1}{2} \cos ^{2} \angle a b}{\frac{1}{2}}=\cos ^{2} \angle a b
\end{gathered}
$$

Note that the reference class used in calculating $C h_{q}\left(e_{A}\right)$ is narrower: it is further restricted by specification of the outcome as of type $V_{B}$ in region $\mathbf{2}$.

Any agent who accepts quantum theory and is (momentarily) located at space-time point $x$ should match credence in $e_{A}$ to $C h_{x}\left(e_{A}\right)$ because it is precisely the role of chance to reflect the epistemic bearing of all information accessible at $x$ on facts not so accessible, and to accept quantum theory is to treat

\footnotetext{
${ }^{5}$ See Ismael [2008]. I have slightly altered her notation to avoid conflict with my own. Here ' $e$ ' ambiguously denotes both an event and the proposition that it occurs. $\mathrm{Cr}$ stands for credence: an agent's degree of belief in a proposition, represented on a scale from 0 to 1 and required to conform to the standard axioms of probability.
} 
Figure 2:

it as an expert when assessing the chances. This is so whether or not an agent is actually located at $x$-fortunately, since it is obviously a gross idealization to locate the epistemic deliberations of a physically situated agent at a space-time point! A hypothetical agent located at $q$ in the forward light cone of region $\mathbf{2}$ (but not 1) has access to the additional information that the outcome in $\mathbf{2}$ is of type $V_{B}$ : so the reference class used to infer the chance of $e_{A}$ at $q$ from the Born rule should include that information. That is why $C h_{q}\left(e_{A}\right)$ is determined by the conditional Born probability $\operatorname{Pr}_{a, b}^{\Phi^{+}}\left(V_{A} \mid V_{B}\right)$ but $C h_{p}\left(e_{A}\right)$ is determined by the unconditional Born probability $\operatorname{Pr}_{a, b}^{\Phi^{+}}\left(V_{A}\right)$.

In the special case that the settings $a, b$ coincide (the polarizers are perfectly aligned) application of the Born rule yields the chances depicted in Figure 3.

Bell ([2004, pp. 240-41]) took this example as a simple demonstration that ordinary quantum mechanics is not locally causal, crediting the argument of EPR [1935]. He begins

Each of the counters considered separately has on each repetition of the experiment a $50 \%$ chance of saying 'yes'.

Each of the chances $C h_{p}\left(e_{A}\right), C h_{p^{\prime}}\left(e_{B}\right)$ is $\frac{1}{2}$, as Bell says: but $C h_{q}\left(e_{A}\right)=1$. After noting that quantum theory here requires a perfect correlation between the outcomes in $\mathbf{1}, \mathbf{2}$, he continues

"So specification of the result on one side permits a $100 \%$ confident prediction of the previously totally uncertain result on the other side. 
Figure 3:

Now in ordinary quantum mechanics there just is nothing but the wavefunction for calculating probabilities. There is then no question of making the result on one side redundant on the other by more fully specifying events in some space-time region $\mathbf{3}$. We have a violation of local causality."

It is true that (Factorizability and (Factorizability ${ }_{S U}$ fail here, since $\operatorname{Pr}_{a, a}^{\Phi^{+}}\left(V_{A} \mid V_{B}\right)=1, \operatorname{Pr}_{a, a}^{\Phi^{+}}\left(H_{A} \mid V_{B}\right)=0$, while $\operatorname{Pr}_{a, a}^{\Phi^{+}}(A)=\operatorname{Pr}_{a, a}^{\Phi^{+}}(B)=\frac{1}{2}$. But does that constitute a violation of local causality? (LC) and $\left(\mathrm{LC}_{S U}\right)$ are both conditions straightforwardly applicable to a theory whose general probabilities yield a unique chance for each possible outcome in $\mathbf{1}$ prior to its occurrence. In the case of quantum theory, however, the general Born rule probabilities yield multiple chances for each possible outcome in $\mathbf{1}$, each at the same time (in the laboratory frame): $C h_{p}\left(e_{A}\right)=\frac{1}{2}$, but $C h_{q}\left(e_{A}\right)=1$ (assuming the outcome in 2 is of type $\left.V_{B}\right)$. When (LC) speaks of "the probabilities attached to $\left[e_{A}, \bar{e}_{A}\right]$ in a space-time region 1 being unaltered by specification of $\left[V_{B}\right]$ in a space-like separated region 2" (my italics), which probabilities are these?

Since the connection to (IP)'s motivating talk of 'cause' and 'effect' is provided by the thought that a cause alters the chance of its effect, (LC) is motivated only if applied to the chances of $e_{A}, \bar{e}_{A}$ in region 1 . But $C h_{p}\left(e_{A}\right)$ is not altered by specification of $V_{B}$ in space-like separated region 2 : its value depends only on what happens in the backward light cone of $\mathbf{1}$, in conformity to its role in prescribing $C r_{p}\left(e_{A}\right)$. Of course $C h_{q}\left(e_{A}\right)$ does depend on the outcome in $\mathbf{2}$. If 
it did not, it could not fulfill its constitutive role of prescribing $C r_{q}\left(e_{A} / I_{q}\right)$ no matter what information $I_{q}$ provides about the contents of $q$ 's past light cone. It follows that $C h_{q}\left(e_{A}\right)$ is not altered but specified by specification of the result in 2.

Only for a hypothetical agent whose world-line has entered the future light cone of $\mathbf{2}$ at $q$ is it true that specification of the result in $\mathbf{2}$ permits a $100 \%$ confident prediction of the previously totally uncertain result on the other side. A hypothetical agent at $p$ is not in a position to make a $100 \%$ confident prediction: for such an agent the result in $\mathbf{1}$ remains totally uncertain: what happens in $\mathbf{2}$ makes no difference to what (s)he should believe, since region $\mathbf{2}$ is outside the backward light cone of $p$. That is why it is $C h_{p}\left(e_{A}\right)$, not $C h_{q}\left(e_{A}\right)$, that says what is certain at $p$. Newtonian absolute time fostered the illusion of the occurrence of future events becoming certain for everyone at the same timewhen they occur if not sooner. Relativity requires certainty, like chance, to be relativized to space-time points - idealized locations of hypothetical knowers.

So does ordinary quantum mechanics violate local causality? If "the probabilities" (LC) speaks of are $\operatorname{Pr}_{a, b}^{\Phi^{+}}\left(V_{A}\right), \operatorname{Pr}_{a, b}^{\Phi^{+}}\left(H_{A}\right)$, and the condition that these be unaltered is understood to be that $\operatorname{Pr}_{a, b}^{\Phi^{+}}\left(V_{A}\right)=\operatorname{Pr}_{a, a}^{\Phi^{+}}\left(V_{A} \mid B\right)$, $\operatorname{Pr}_{a, b}^{\Phi^{+}}\left(H_{A}\right)=\operatorname{Pr}_{a, a}^{\Phi^{+}}\left(H_{A} \mid B\right)$, then ordinary quantum mechanics violates (LC). But if this is all (LC) means, then it is not motivated by (IP) and its violation does not imply that the quantum world is non-local in that there are superluminal causal relations between distant events. For (LC) to be motivated by causal considerations such as (IP), "the probabilities" (LC) speaks of must be understood to be chances, including $C h_{p}\left(e_{A}\right)$ and $C h_{q}\left(e_{A}\right)$. But neither of these would be altered by the specification of the outcome $\left[V_{B}\right]$ in a space-like separated region, so local causality would then not be violated. Although it remains unclear exactly how (LC) (or $\left(\mathrm{LC}_{S U}\right)$ ) is supposed to be applied to quantum mechanics, one way of applying it is unmotivated by (IP), while if it is applied in another way quantum mechanics does not violate this local causality condition.

\section{Chance and Causation}

Suppose in the EPR-Bohm scenario that the outcome in region $\mathbf{2}$ had been of type $H_{B}$ instead of $V_{B}$ : then $C h_{q}\left(e_{A}\right)$ would have been 0 instead of 1 . Suppose that the polarization axis for the measurement in region $\mathbf{2}$ had been rotated through $60^{\circ}$ : then $C h_{q}\left(e_{A}\right)$ would have been $\frac{1}{4}$ or $\frac{3}{4}$, depending on the outcome in region 2. Or suppose that no polarization measurement had been performed in region 2: then $C h_{q}\left(e_{A}\right)$ would have been $\frac{1}{2}$. This shows that $C h_{q}\left(e_{A}\right)$ depends counterfactually on the polarization measurement in $\mathbf{2}$ and also on its outcome. Another way to understand talk of "alteration" of "the" probability of an event of type $V_{A}$ is as the difference between the actual value of $C h_{q}\left(e_{A}\right)$ and what its value would have been had the polarization measurement in $\mathbf{2}$ or its outcome been different. Don't such counterfactual "alterations" in $C h_{q}\left(e_{A}\right)$ amount to causal dependence between space-like separated events, in violation of (IP)? 
There are several reasons why they do not.

The first reason is that while $C h_{q}\left(e_{A}\right)$ would be different in each of these counterfactual scenarios, in none of them would $C h_{p}\left(e_{A}\right)$ differ from $\frac{1}{2}$, so the "local" chance of $e_{A}$ is insensitive to all such counterfactual variations in what happens in 2. If one wishes to infer causal from counterfactual dependence of "the" chance of a result in $\mathbf{1}$ on what happens in $\mathbf{2}$, then only one of two relevant candidates for "the" chance displays such counterfactual dependence. For those who think of chance as itself a kind of indeterministic cause - a localized physical propensity whose actualization may produce an effect $-C h_{p}\left(e_{A}\right)$ seems better qualified for the title of "the" chance of $e_{A}$ than $C h_{q}\left(e_{A}\right)$.

The role of chance in decision provides the second reason. Just as the chance of $e$ tells you everything you need to know to figure out how strongly to believe $e$, the causal dependence of $e$ on $d$ tells you everything you need to know about $e$ and $d$ when deciding whether to do $d$ (assuming you are not indifferent about $e$ ). As Huw Price [2012] put it, "causal dependence should be regarded as an analyst-expert about the conditional credences required by an evidential decision maker".

Consider the situation of a hypothetical agent Bob at $p^{\prime}$ deciding whether to act by affecting what happens in $\mathbf{2}$ to try to get outcome $e_{A}$ in $\mathbf{1}$. Bob can choose not to measure anything, or he can choose to measure polarization with respect to any axis $b$. If he were to measure nothing, $C h_{q}\left(e_{A}\right)$ would be $\frac{1}{2}$. If he were to measure polarization with respect to the same axis as Alice, then $C h_{q}\left(e_{A}\right)$ would be either 0 or 1 , with an equal chance (at his momentary location $p^{\prime}$ ) of either outcome. Since he can neither know nor affect which of these chances it will be, he must base his decision on his best estimate of $C h_{q}\left(e_{A}\right)$ in accordance with Ismael's [2008] Ignorance Principle:

"Where you're not sure about the chances, form a mixture of the chances assigned by different theories of chance with weights determined by your relative confidence in those theories."

Following this principle, Bob should assign $C h_{q}\left(e_{A}\right)$ the estimated value $\frac{1}{2} \cdot 0+\frac{1}{2} \cdot 1=\frac{1}{2}$, and base his decision on that. Since measuring polarization with respect to the same axis as Alice would not raise his estimated chance of securing outcome $e_{A}$ in $\mathbf{1}$, he should eliminate this option whether or not he could execute it. His estimated value of $C h_{q}\left(e_{A}\right)$ were he to measure polarization with respect to an axis rotated $60^{\circ}$ from Alice's is also $\frac{1}{2}\left(\frac{1}{2} \cdot \frac{1}{4}+\frac{1}{2} \cdot \frac{3}{4}=\frac{1}{2}\right)$. Similarly for any other angle. This essentially recapitulates part of the content of the no-signalling theorems, going back to Eberhard [1978]. Bell [2004, pp. 237-8] shows why manipulation of external fields at $p^{\prime}$ or in $\mathbf{2}$ would also fail to alter Bob's estimated value of $C h_{q}\left(e_{A}\right)$.

But what if Bob had simply arranged for the measurement in $\mathbf{2}$ to have had the different outcome $\bar{e}_{B}$ ? Then $C h_{q}\left(e_{A}\right)$ would have been 0 instead of 1 . No-one who accepts quantum mechanics can countenance this counterfactual scenario. The Born rule implies that $\operatorname{Pr}_{b}^{\Phi^{+}}\left(H_{B}\right)=\frac{1}{2}$, and anyone who accepts quantum mechanics accepts the implication that $C h_{p^{\prime}}\left(\bar{e}_{B}\right)=\frac{1}{2}$. So anyone who accepts 
quantum mechanics will have credence $C r_{p^{\prime}}\left(\bar{e}_{B} / I_{p^{\prime}}\right)=\frac{1}{2}$ no matter what he takes to happen in the backward light cone of $p^{\prime}$ (as specified by $I_{p^{\prime}}$ ) 6 If he accepts quantum mechanics, Bob will conclude that there is nothing it makes sense to contemplate doing to alter his estimate of $C h_{p^{\prime}}\left(\bar{e}_{B}\right)$, and so there is no conceivable counterfactual scenario in which one in Bob's position arranges for the measurement in $\mathbf{2}$ to have had the different outcome $\bar{e}_{B}$. In general, there is causal dependence between events in $\mathbf{1}$ and $\mathbf{2}$ only if it makes sense to speak of an intervention in one of these regions that would affect a hypothetical agent's estimated chance of what happens in the other. Anyone who accepts quantum mechanics should deny that makes sense.

Perhaps the most basic reason why counterfactual dependencies between happenings in region 2 and the chance(s) of $e_{A}$ are no sign of causal dependence is that chances are not beables, and are incapable of entering into causal relations. That Bell thought they behaved like beables is suggested by the [1975] paper in which he introduced local causality as a natural generalization of local determinism:

"In Maxwell's theory, the fields in any space-time region $\mathbf{1}$ are determined by those in any space region $V$, at some time $t$, which fully closes the backward light cone of $\mathbf{1}$. Because the region $V$ is limited, localized, we will say the theory exhibits local determinism. We would like to form some no[ta]tion of local causality in theories which are not deterministic, in which the correlations prescribed by the theory, for the beables, are weaker." [2004, p. 53]

It seems Bell thought the chances prescribed by a theory that is not deterministic were analogous to the beables of Maxwell's electromagnetism, so that while local determinism (locally) specified the local[ized] beables (e.g. fields), local causality should (locally) specify the local[ized] chances of beables, where those chances (like local beables) are themselves localized physical magnitudes.

Others have joined Bell in this view of chances as localized physical magnitudes. But quantum mechanics teaches us that chances are not localized physical propensities whose actualization may produce an effect. Maudlin says what he means by calling probabilities objective:

"...there could be probabilities that arise from fundamental physics, probabilities that attach to actual or possible events in virtue solely of their physical description and independent of the existence of cognizers. These are what I mean by objective probabilities." (Beisbart and Hartmann eds., [2011, p. 294])

Although quantum chances do attach to actual or possible events, they are not objective in this sense. As we saw, the chance of outcome $e_{A}$ does not attach to it in virtue solely of its physical description: the chances of $e_{A}$ attach also

\footnotetext{
${ }^{6} \mathrm{~A}$ unitary evolution $\Phi^{+} \Rightarrow \Xi^{+}$corresponding to a local interaction there would still yield $\operatorname{Pr}_{b}^{\Xi^{+}}\left(H_{B}\right)=\frac{1}{2}$.
} 
in virtue of its space-time relations to different space-time locations. Each such location offers the epistemic perspective of a situated agent, even in a world with no such agents. The existence of these chances is independent of the existence of cognizers. But it is only because we are not merely cognizers but physically situated agents that we have needed to develop a concept of chance tailored to our needs as informationally deprived agents. Quantum chance admirably meets those needs: an omniscient God could describe and understand the physical world without it.

While they are neither physical entities nor physical magnitudes, quantum chances are objective in a different sense. They supply an objective prescription for the credences of an agent in any physical situation. Anyone who accepts quantum mechanics is committed to following that prescription.

\section{A view of quantum mechanics}

As I see it [2012], it is not the function of quantum states, observables, probabilities or the Schrödinger equation to represent or describe the condition or behavior of a physical system to which they are associated. These elements function in other ways when a quantum model is applied in predicting or explaining physical phenomena such as non-localized correlations. Assignment of a quantum state may be viewed as merely the first step in a procedure that licenses a user of quantum mechanics to express claims about physical systems in descriptive language and then warrants that user in adopting appropriate epistemic attitudes toward some of these claims. The language in which such claims are expressed is not the language of quantum states or operators, and the claims are not about probabilities or measurement results: they are about the values of physical magnitudes, and I'll refer to them as magnitude claims. Magnitude claims were made by physicists and others before the development of quantum mechanics and continue to be made, some in the same terms, others in terms newly introduced as part of some scientific advance. But even though quantum mechanics represents an enormous scientific advance, claims about quantum states, operators and probability distributions are not magnitude claims.

The quantum state has two roles. One is in the algorithm provided by the Born Rule for assigning probabilities to significant claims of the form $M_{\Delta}(s)$ : The value of $M$ on $s$ lies in $\Delta$, where $M$ is a physical magnitude, $s$ is a physical system and $\Delta$ is a Borel set of real numbers. In what follows, I will call a descriptive claim of the form $M_{\Delta}(s)$ a canonical magnitude claim. For two such claims the formal algorithm may be stated as follows:

$$
\operatorname{Pr}\left(M_{\Delta}(s), N_{\Gamma}(s)\right)=\operatorname{Tr}\left(\rho \hat{P}^{M}[\Delta] \cdot \hat{P}^{N}[\Gamma]\right) \quad \text { (Born Rule) }
$$

Here $\rho$ represents a quantum state as a density operator on a Hilbert space $\mathcal{H}_{s}$ and $\hat{P}^{M}[\Delta]$ is the value for $\Delta$ of the projection-valued measure defined by the unique self-adjoint operator on $\mathcal{H}_{s}$ corresponding to $M$.

But the significance of a claim like $M_{\Delta}(s)$ varies with the circumstances to which it relates. Accordingly, a quantum state plays a second role by mod- 
ulating the content of $M_{\Delta}(s)$ or any other magnitude claim by modifying its inferential relations to other claims. Because I believe the nature of this modulation of content renders inappropriate the metaphor of magnitudes corresponding to elements of reality, I recommend against thinking of magnitudes that figure in canonical or other magnitude claims as beables, even though many such magnitude claims are true. But if one insists on calling magnitudes that figure in magnitude claims beables, these magnitudes are not beables introduced by quantum mechanics - they are at most beables recognized in its applications 7

The quantum state is not a beable in this view. Indeed, since none of the distinctively quantum elements of a quantum model qualifies as a beable introduced by the theory, quantum mechanics has no beables of its own. Viewed this way, a quantum state does not describe or represent some new element of physical reality 8 But nor is it the quantum state's role to describe or represent the epistemic state of any actual agent. A quantum state assignment is objectively true (or false): in that deflationary sense a quantum state is objectively real. But its function is not to say what the world is like but to help an agent applying quantum mechanics to predict and explain what happens in it. It is physical conditions in the world that make a quantum state assignment true (or false). True quantum state assignments are backed by true magnitude claims, though some of these are typically about physical systems other than that to which the state is assigned.

Any application of quantum mechanics involves claims describing a physical situation. While it is considered appropriate to make claims about where individual particles are detected contributing to the interference pattern in a contemporary interference experiment, claims about through which slit each particle went are frequently alleged to be "meaningless". In its second role the quantum state offers guidance on the inferential powers, and hence the content, of canonical magnitude claims.

The key idea here is that even assuming unitary evolution of a joint quantum state of system and environment, delocalization of system state coherence into the environment will typically render descriptive claims about experimental outcomes and the condition of apparatus and other macroscopic objects appropriate by endowing these claims with enough content to license an agent to adopt epistemic attitudes toward them, and in particular to apply the Born Rule. But an application of quantum mechanics to determine whether this is so will not require referring to any system as "macroscopic", as an "apparatus" or as an "environment". All that counts is how a quantum state of a super-system evolves in a model, given a Hamiltonian associated with an interaction between the system of interest and the rest of that super-system.

It is important to note that since the formulation of the Born Rule now involves no explicit or implicit reference to "measurement", Bell's ([2004, pp. 213-31]) strictures against the presence of the term 'measurement' in a precise formulation of quantum mechanics are met. None of the other proscribed terms

\footnotetext{
${ }^{7}$ Compare Bell [2004, p. 55].

${ }^{8}$ Compare Bell [2004, p. 53]: "...this does not bother us if we do not grant beable status to the wave-function."
} 
'classical', 'macroscopic', 'irreversible', or 'information' appears in its stead.

Since an agent's assignment of a quantum state does not serve to represent a system's properties, her reassignment of a "collapsed" state on gaining new information represents no change in that system's properties. That is why collapse is not a physical process, in this view of quantum mechanics. Nor does the Schrödinger equation express a fundamental physical law: to assign a quantum state to a system is not to represent its dynamical properties. A formulation of quantum mechanics has no need to include a statement distinguishing the circumstances in which physical processes of Schrödinger evolution and "collapse" occur. An agent can use quantum mechanics to track changes of the dynamical properties of a system by noting what magnitude claims are significant and true of it at various times. But quantum mechanics itself does not imply any such claim, even when an agent would be correct to assign a system a quantum state, appropriately apply the Born Rule, and conclude that the claim has probability 1 .

Quantum states are relational on this interpretation. When agents (actually or merely hypothetically) occupy relevantly different physical situations they should assign different quantum states to one and the same system, even though these different quantum state assignments are equally correct. The primary function of Born probabilities is to offer a physically situated agent authoritative advice on how to apportion degrees of belief concerning contentful canonical magnitude claims that the agent is not currently in a position to check. That is why the Born rule should be applied by differently situated agents to assign different chances to a single canonical magnitude claim $M_{\Delta}(s)$ about a system $s$ in a given situation. These different chance assignments will then be equally objective and equally correct.

The physical situation of a (hypothetical or actual) agent will change with (local) time. The agent may come to be in a position to check the truthvalues of previously inaccessible magnitude claims, some of which may be taken truly to describe outcomes of measurements. If a quantum state is to continue to provide the agent with good guidance concerning still inaccessible magnitude claims, it must be updated to reflect these newly accessible truths. The required alteration in the quantum state is not a physical process involving the system, in conflict with Schrödinger evolution. What has changed is just the physical relation of the agent to events whose occurrence is described by true magnitude claims. This is not represented by a discontinuous change in the quantum state of some model: it corresponds to adoption of a new quantum model that incorporates additional information, newly accessible to the user of quantum mechanics.

The preceding paragraphs contained a lot of talk of agents. To forestall misunderstandings, I emphasize that quantum mechanics is not about agents or their states of knowledge or belief: A precise formulation of quantum mechanics will not speak of such things in its models any more than it will speak of agents' measuring, observing or preparing activities. If quantum mechanics is about anything it is about the quantum systems, states, observables and probability measures that figure in its models. Quantum mechanics, like all scientific the- 
ories, was developed by (human) agents for the use of agents (not necessarily human: while insisting that any agent be physically situated, I direct further inquiry on the constitution of agents to cognitive scientists). Trivially, only an agent can apply a theory for whatever purpose. So any account of a predictive, explanatory or other application of quantum mechanics naturally involves talk of agents. 


\section{How to use quantum mechanics to explain non-localized correlations}

In his [1981] Bell argued that

certain particular correlations, realizable according to quantum mechanics, are locally inexplicable. They cannot be explained, that is to say, without action at a distance. [2004, pp. 151-2]

The particular correlations to which Bell refers arise, for example, in the EPR-Bohm scenario in which pairs of spin $\frac{1}{2}$ "particles" are prepared in a singlet spin state, then at widely separated locations each element of a pair is passed through a Stern-Gerlach magnet and detected either in the upper or in the lower part of a screen. By calling them realizable rather than realized he acknowledged the experimental difficulties associated with actually producing statistics supporting them in the laboratory (or elsewhere). Enormous improvements in experimental technique since 1981 have overcome most of the difficulties associated with performing a "loophole-free" test of CHSH or other so-called Bell inequalities and at the same time provided very strong statistical evidence for quantum mechanical predictions in analogous experiments. Since the improvements have been most dramatic for experiments involving polarization measurements on entangled photons, it is appropriate to refer back to the experimental scenario discussed by Bell in 1990 ([2004, pp. 232-248]).

Suppose photon pairs are prepared at a central source in the entangled polarization state $\Phi^{+}=1 / \sqrt{2}(|H H\rangle+|V V\rangle)$, and the photons in a pair are both subsequently detected in coincidence at two widely separated locations after each has passed through a polarizing beam splitter (PBS) with axis set at $a, b$ respectively. If a photon is detected with polarization parallel to this axis, a macroscopic record signifies "yes": if it is detected with polarization perpendicular to this axis, the record signifies "no". Let the record "yes" at one location be the event of a magnitude $A$ taking on value +1 , "no" the event of $A$ taking on value -1 , and similarly for $B$ at the other location. Let $a$ be a locally generated signal that quickly sets the axis of the PBS on the $A$ side to an angle $a^{\circ}$ from some standard direction, and similarly for $b$ on the $B$ side. Assume this is done so that each of $a$ and $A$ 's taking on a value is space-like separated from each of $b$ and $B$ 's taking on a value. In this scenario quantum mechanics predicts that, for $a^{\circ}=0^{\circ}, a^{\circ}=45^{\circ}, b^{\circ}=22 \frac{1}{2}^{\circ}, b^{\circ}=-22 \frac{1}{2}^{\circ}$

$$
E(a, b)+E\left(a, b^{\prime}\right)+E\left(a^{\prime}, b\right)-E\left(a^{\prime}, b^{\prime}\right)=2 \sqrt{2}
$$

where, for example, $E(a, b) \equiv \operatorname{Pr}_{a, b}(+1,+1)+\operatorname{Pr}_{a, b}(-1,-1)-\operatorname{Pr}_{a, b}(+1,-1)-$ $\operatorname{Pr}_{a, b}(-1,+1)$. This is in violation of the CHSH inequality

$$
\left|E(a, b)+E\left(a, b^{\prime}\right)+E\left(a^{\prime}, b\right)-E\left(a^{\prime}, b^{\prime}\right)\right| \leq 2
$$

that follows from $($ Factorizability $S U)$. Bell claims these correlations are realizable according to quantum mechanics but that they cannot be explained without 
action at a distance. While it is generally acknowledged that quantum mechanics successfully predicts Bell's particular correlations, demonstrating this will illustrate the present view of quantum mechanics. To decide whether it also explains them we need to ask what more is required of an explanation.

What we take to be a satisfactory explanation has changed during the development of physics, and we may confidently expect such change to continue. One who accepts quantum mechanics is able to offer a novel kind of explanation. Nevertheless, explanations of phenomena using quantum mechanics may be seen to meet two very general conditions met by many, if not all, good explanations in physics.

(i) They show that the phenomenon to be explained was to be expected, and (ii) they say what it depends on.

Quantum mechanics enables us to give explanations meeting both conditions.

Meeting the first condition is straightforward. Anyone accepting quantum mechanics can use the Born Rule applied to state $\Phi^{+}$to calculate joint probabilities such as $\operatorname{Pr}_{a, b}(A, B)$ and go on to derive (7). So for anyone who accepts quantum mechanics, violation of the CHSH inequalities is to be expected. But it is worth showing in more detail how quantum mechanics can be applied to derive (7) because this will help to exhibit the relational nature of quantum states and probabilities while making it clear that a precise formulation of quantum mechanics need not use the word 'measurement' or any other term on Bell's list of proscribed words [2004, p. 215].

Figure 4: 
Figure 4 is a space-time diagram depicting space-like separated polarization measurements by Alice and Bob in regions 1,2 respectively on a photon pair. Time is represented in the laboratory frame. At $t_{1}$ each takes the polarization state of the $L-R$ photon pair to be $\Phi^{+}=1 / \sqrt{2}(|H H\rangle+|V V\rangle)$. What justifies this quantum state assignment is their knowledge of the conditions under which the photon pair was produced-perhaps by parametric down-conversion of laser light by passage through a non-linear crystal. Such knowledge depends on information about the physical systems involved in producing the pair. This state assignment is backed by significant magnitude claims about such systems - if anything counts as a claim about beables recognized by quantum mechanics, these do. Then Alice measures polarization of photon $L$ along axis $a^{\circ}$, Bob measures polarization of photon $R$ along axis $b^{\circ}$. Decoherence at the photon detectors licenses both of them to treat the Born rule measure corresponding to state assignment $\Phi^{+}$as a probability distribution over significant canonical magnitude claims about the values of $A, B$.

At $t_{1}$ Alice and Bob should both assign state $\Phi^{+}$and apply the Born Rule to calculate the joint probability $\operatorname{Pr}_{a, b}^{\Phi^{+}}(A, B)$ assigned to claims about the values of magnitudes $A, B$ - claims that we may, but need not, choose to describe as records of polarization measurements along both $a^{\circ}, b^{\circ}$ axes - and hence the (well-defined) conditional probability $\operatorname{Pr}_{a, b}^{\Phi^{+}}(A, B) / \operatorname{Pr}_{a, b}^{\Phi^{+}}(B)=|\langle A \mid B\rangle|^{2}$. Each will then expect the observed non-localized correlations between the outcomes of polarization measurements in regions 1,2 when the detectors are set along the $a^{\circ}, b^{\circ}$ axes. They will expect analogous correlations as these axes are varied, and so they will expect (7) (violating the CHSH inequality) in such a scenario.

At $t_{2}$, after recording polarization $V_{B}$ for $R$, Bob should assign pure state $\left|V_{B}\right\rangle$ to $L$ and use the Born Rule to calculate the probabilities $\operatorname{Pr}_{a}^{\left|V_{B}\right\rangle}(A)=$ $\left|\left\langle A \mid V_{B}\right\rangle\right|^{2}$ for Alice to record polarization of $L$ with respect to the $a^{\circ}$-axis. At $t_{2}$, Alice should assign state $\hat{\rho}=\frac{1}{2} \hat{1}$ to $L$, and use the Born Rule to calculate probability $\operatorname{Pr}_{a}^{\rho}(A)=\frac{1}{2}$ that she will record either polarization of $L$ with respect to the $a^{\circ}$-axis. In this way each forms expectations as to the outcome of Alice's measurement on the best information available to him or her at $t_{2}$. Alice's statistics of her outcomes in many repetitions of the experiment are just what her quantum state $\frac{1}{2} \hat{1}$ for $L$ led her to expect, thereby helping to explain her results. Bob's statistics for Alice's outcomes (in many repetitions in which his outcome is $V_{B}$ ) are just what his quantum state $\left|V_{B}\right\rangle$ for $L$ led him to expect, thereby helping him to explain Alice's results.

There is no question as to which, if either, of the quantum states $\left|V_{B}\right\rangle$, $\frac{1}{2} \hat{1}$ was the real state of Alice's photon at $t_{2}$. Neither of the different probabilities $\left|\left\langle A \mid V_{B}\right\rangle\right|^{2}$ or $\frac{1}{2}$ represents a unique physical propensity at $t_{2}$ of Alice's outcomeeven though neither its chance at $p$ nor its chance at $q$ is subjective. This discussion applies independent of the time-order in Alice's frame of the regions 1, 2 in Figure 1: had she been moving away from Bob fast enough, she would have represented 1 as earlier than 2 .

It is widely acknowledged that one cannot explain a phenomenon merely by showing that it was to be expected in the circumstances. To repeat a hack- 
neyed counterexample, the falling barometer does not explain the coming storm even though it gives one reason to expect a storm in the circumstances. As a joint effect of a common cause, a symptom does not explain its other independent effects. But a system's being in a quantum state at a time is not a symptom of causes specified by the true magnitude claims that back it since it is not an event distinct from the conditions those claims describe. Each event figuring in Bell's particular correlations is truly described by a canonical magnitude claim. We may choose to describe some, but not all, such events as an outcome of a quantum measurement on a system: the probabilities of many of those events depend counterfactually on the particular entangled state assigned at $t_{1}$-if that state had been different, so would these probabilities. But this dependence is not causal. In quantum mechanics, neither states nor probabilities are the sorts of things that can bear causal relations: in Bell's terminology, they are not beables.

When relativized to the physical situation of an actual or hypothetical agent, a quantum state assignment is objectively true or false - which depends on the state of the world. More specifically, a quantum state assignment is made true by the true magnitude claims that back it. One true magnitude claim backing the assignment of $\left|V_{B}\right\rangle$ to $L$ at $q$ reports the outcome of Bob's polarization measurement in region 2 of Figure 1: but there are others, since this would not have been the correct assignment had the correct state assignment at $p^{\prime}$ been $\left|H_{A}\right\rangle\left|V_{B}\right\rangle$. We also need to ask for the backing of the entangled state $\Phi^{+}$.

There are many ways of preparing state $\Phi^{+}$, and this might also be the right state to assign to some naturally occurring photon pairs that needed no preparation. In each case there is a characterization in terms of some set of true magnitude claims describing the systems and events involved: these back the state assignment $\Phi^{+}$. It may be difficult or even impossible to give this characterization in a particular case, but that is just an epistemic problem which need not be solved even by experimenters skilled in preparing or otherwise assigning this state. $\Phi^{+}$will be correctly assigned at $p^{\prime}$ only if some set of true magnitude claims backing that assignment is accessible from $p^{\prime}$ : events making them true must lie in the backward light-cone of $p^{\prime}$.

A quantum state counterfactually depends on the true magnitude claims that back it in somewhat the same way that a dispositional property depends on its categorical basis. The state $\Phi^{+}$may be backed by alternative sets of true magnitude claims just as a person may owe his immunity to smallpox to any of a variety of categorical properties. If Walt owes his smallpox immunity to antibodies, his possession of antibodies does not cause his immunity: it is what his immunity consists in. No more is the state $\Phi^{+}$caused by its backing magnitude claims: a statement assigning state $\Phi^{+}$is true only if backed by some true magnitude claims of the right kind. A quantum state is counterfactually dependent on whatever magnitude claims back it because backing is a kind of determination or constitution relation, not because it is a causal relation.

In this view, a quantum state causally depends neither on the physical situation of the (hypothetical or actual) agent assigning it nor on any of its 
backing magnitude claims. The correct state $\left|V_{B}\right\rangle$ to be assigned to $L$ at $q$ is not causally dependent on anything about Bob's physical situation even if he happens to be located at $q$ : it is not causally dependent on the outcome of Bob's polarization measurement in region 2: and it is not causally dependent on how Bob sets his polarizer in region 2. But a quantum state assignment is not just a function of the subjective epistemic state of any agent: If Bob or anyone else were to assign a state other than $\left|V_{B}\right\rangle$ to $L$ at $q$ he or she would be making a mistake.

The quantum derivation of (7) shows not only that Bell's particular correlations were to be expected, but also what they depend on. They depend counterfactually but not causally on the quantum state $\Phi^{+}$, and they also depend counterfactually on that state's backing conditions, as described by true magnitude claims. The status of the quantum state disqualifies it from participation in causal relations, but true magnitude claims may be taken to describe beables recognized by quantum mechanics. To decide which conditions backing any of the states involved in their explanation describe causes of Bell's particular correlations or the events they correlate we need to return to the connection between causation and chance.

The intuition that, other things being equal, a cause raises (or at least alters) the chance of its effect is best cashed out in terms of an interventionist counterfactual: $c$ is a cause of $e$ just in case $c, e$ are distinct actual events and there is some conceivable intervention on $c$ whose occurrence would have altered the chance of $e$. Such an intervention need not be the act of an agent: it could involve any modification in $c$ of the right kind. Woodward [2003, p. 98] is one influential attempt to say what kind of external influence this would involve. Note that Einstein's formulation of a principle of local action also appeals to intervention:

The following idea characterizes the relative independence of objects far apart in space $(A$ and $B)$ : external influence on $A$ has no immediate ("unmittelbar") influence on $B$; this is known as the "principle of local action' (Einstein [1948, pp. 321-2])

I used the idea of intervention to argue against any causal dependence between events in $\mathbf{1}$ and 2: anyone who accepts quantum mechanics accepts that it makes no sense to speak of an intervention in one of these regions that would affect a hypothetical agent's estimated chance of what happens in the other. So even though the outcome $e_{B}$ in $\mathbf{2}$ backs the assignment $\left|V_{B}\right\rangle$ to $L$ at $q$, the outcome in $\mathbf{1}$ does not depend causally on $e_{B}$ : for similar reasons, neither does the outcome in $\mathbf{2}$ depend on that in $\mathbf{1}$. The same idea can now be used to show that both these outcomes do depend causally on whatever event $o$ in the overlap of the backward light cones of $\mathbf{1}$ and $\mathbf{2}$ warranted assignment of state $\Phi^{+}$-an event truly described by magnitude claims that backed this assignment.

Assume first that the events $a, b$ at which the polarizers are set on a particular occasion occur in the overlap of the backward light cones of $\mathbf{1}$ and $\mathbf{2}$ : this assumption will later be dropped. Let $r$ be a point outside the future light 
cones of $e_{A}, e_{B}$ but within the future light cone of the event $o$. Let $e_{A} \uplus e_{B}$ be the event of the joint occurrence of $e_{A}, e_{B}$. This is an event of a type to which the Born rule is applicable: the application yields its chance $C h_{r}\left(e_{A} \uplus e_{B}\right)=$ $\operatorname{Pr}_{a, b}^{\Phi^{+}}\left(V_{A}, V_{B}\right)=\frac{1}{2} \cos ^{2} \angle a b$. We already saw that $C h_{r}\left(e_{A}\right)=\operatorname{Pr}_{a, b}^{\Phi^{+}}\left(V_{A}\right)=\frac{1}{2}=$ $\operatorname{Pr}_{a, b} \Phi^{+}\left(V_{B}\right)=C h_{r}\left(e_{B}\right)$. The event $o$ affects all these chances: had a different event $o^{\prime}$ occurred backing the assignment of a different state (e.g. $\left|H_{A}\right\rangle\left|V_{B}\right\rangle$ ), or no event backing any state assignment, then any or all of these chances could have been different. Since it makes sense to speak of an agent altering the chance of event $o$ at $s$ in its past light cone, we have

$$
\begin{gathered}
C h_{r}\left(e_{A} \uplus e_{B} \mid d o-o\right) \neq C h_{r}\left(e_{A} \uplus e_{B}\right) \\
C h_{r}\left(e_{A} \mid d o-o\right) \neq C h_{r}\left(e_{A}\right) \\
C h_{r}\left(e_{B} \mid d o-o\right) \neq C h_{r}\left(e_{B}\right)
\end{gathered}
$$

where $d o-o$ means $o$ is the result of an intervention without which $o$ would not have occurred. It follows that $e_{A}, e_{B}, e_{A} \uplus e_{B}$ are each causally dependent on $o: o$ is a common cause of $e_{A}, e_{B}$ even though the probabilities of events of these types do not factorize. The same reasoning applies to each registered photon pair on any occasion at any settings $a, b$. So the second requirement on explanation is met: the separate recording events, as well as the event of their joint occurrence, depend causally on the event $o$ that serves to back assignment of state $\Phi^{+}$to the photon pairs involved in this scenario.

By rejecting any possibility of an intervention expressed by $d o-e_{B}$ or $d o-\bar{e}_{B}$, anyone accepting quantum mechanics should deny that $C h_{p(q)}\left(e_{A} \mid d o-\right.$ $\left.e_{B}\right) \neq C h_{p(q)}\left(e_{A} \mid d o-\bar{e}_{B}\right)$ is true or even meaningful. Nevertheless $C h_{q}\left(e_{A} \mid e_{B}\right) \neq$ $C h_{q}\left(e_{A} \mid \bar{e}_{B}\right)$ : in this sense $e_{A}$ depends counterfactually but not causally on $e_{B}$. Does such counterfactual dependence provide reason enough to conclude that $e_{A}$ is part of the explanation of $e_{B}$ ? An obvious objection is that because of the symmetry of the situation with $\mathbf{1}$ and $\mathbf{2}$ space-like separated there is an equally strong reason to conclude that $e_{B}$ is part of the explanation of $e_{A}$, contrary to the fundamentally asymmetric nature of the explanation relation. But one can see that this objection is not decisive by paying attention to the contrasting epistemic perspectives associated with the different physical situations of hypothetical agents Alice* and Bob* with world-lines confined to interiors of the light cones of $\mathbf{1}, \mathbf{2}$ respectively.

As his world-line enters the future light cone of $\mathbf{2} \mathrm{Bob}^{*}$ comes into position to know the outcome at $\mathbf{2}$ while still physically unable to observe the outcome at $\mathbf{1}$. His epistemic situation is then analogous to that of a hypothetical agent Chris in a world with a Newtonian absolute time, in a position to know the outcome of past events but physically unable to observe any future event. Many have been tempted to elevate the epistemic asymmetry of Chris's situation into a global metaphysical asymmetry in which the future is open while the past is fixed and settled. It is then a short step to a metaphysical view of explanation as a productive relation in which the fixed past gives rise to the (otherwise) 
open future, either deterministically or stochastically 9

Such a move from epistemology to metaphysics should always be treated with deep suspicion. But in this case it is clearly inappropriate in a relativistic space-time since the "open futures" of agents like Alice* and Bob* cannot be unified into the open future. This prompts a retreat to a metaphysically drained view of explanation as rooted in cognitive concerns of a physically situated agent, motivated by the need to unify, extend and efficiently deploy the limited information to which it has access.

For many purposes it is appropriate to regard the entire scientific community as a (spatially) distributed agent, and to think of the provision of scientific explanations as aiding our collective epistemic and practical goals. This is appropriate insofar as localized agents share an epistemic perspective, with access to the same information about what has happened. But Alice* and Bob* do not have access to the same information at time $t_{2}$ or $t_{3}$ since they are then space-like separated. So it is entirely appropriate for Bob* to use $e_{B}$ to explain $e_{A}$ and for Alice* to use $e_{A}$ to explain $e_{B}$. This does not make explanation a subjective matter for two reasons. There is an objective physical difference between the situations of Alice* and Bob* underlying the asymmetry of their epistemic perspectives: and by adopting either perspective in thought (as I have encouraged the reader to do) anyone can come to appreciate how each explanation can help make Bell's correlations seem less puzzling. Admittedly, neither explanation is very deep, and I will end by noting one puzzle that remains.

By meeting both minimal requirements on explanation, the application of quantum theory enables us to explain Bell's correlations. But is this explanation local? Several senses of locality are relevant here. The explanation involves no superluminal causal dependence. As stated, the condition of Local Causality is not applicable to the quantum mechanical explanation since it presupposes the uniqueness of the probability to which it refers. (Factorizability ${ }_{S U}$ (and presumably also (Factorizability $)$ ) are violated, but Bell ([2004, p. 243]) preferred to see (Factorizability) as not a formulation but a consequence of 'local causality': I have argued that it is not. To retain its connection to (IP), a version of Local Causality should speak of chances rather than general probabilities. A version that equates the unconditional chance of $e_{A}$ to its chance conditional on $e_{B}$ holds, no matter how these chances are relativized to the same spacetime point. But a version that is clearly motivated by the intuitive principle (IP) would rather equate the unconditional chance of $e_{A}$ to its chance conditional on an intervention that produces $e_{B}$. However this version is inapplicable since acceptance of quantum mechanics renders senseless talk of interventions producing $e_{B}$.

The explanation one can give by applying quantum mechanics appeals to chances that are localized, insofar as they are assigned at space-time points that may be thought to offer the momentary perspective of a hypothetical idealized agent whose credences they would guide. But these chances are not quantum beables and they are not physical propensities capable of manifestation at

${ }^{9}$ See, for example, Maudlin [2007, pp. 173-8]. 
those locations (or anywhere else). The only causes figuring in the explanation are localized where the physical systems are whose magnitudes back the assignment of state $\Phi^{+}$. That chances are not propensities becomes clear when one drops the assumption that $a, b$ occur in the overlap of the backward light cones of $\mathbf{1}$ and $\mathbf{2}$, as depicted in Figure 5.

Figure 5:

If $a, b$ are set at the last moment, the chance of $e_{A} \uplus e_{B}$ that figures in its explanation may be located later in the laboratory frame than $e_{A}, e_{B}$. If chance were a physical propensity it should act before its manifestation. But chances aren't propensities - proximate causes of localized events. They are a localized agent's objective guide to credence about epistemically inaccessible events.

I will conclude by noting one sense in which the explanation one can give using quantum mechanics is not local as it stands. Though it is a (nonfactorizable) cause of events of types $A, B$ in regions $\mathbf{1}, \mathbf{2}$ respectively, the event $o$ is not connected to its effects by any spatiotemporally continuous causal process described by quantum mechanics. This puts the explanation in tension with the first conjunct of Bell's ([2004, p. 239]) intuitive locality principle (IP): "The direct causes (and effects) of events are near by, and even the indirect causes (and effects) are no further away than permitted by the velocity of light."

$o$ is separated from both recording events in regions 1, $\mathbf{2}$ in time, and from at least one in space. If $o$ is not merely a cause but a direct cause of these events then it violates the first conjunct of (IP) because it is not nearby. But if one adopts the present view of quantum mechanics, the theory has no resources to describe any causes mediating between $o$ and these recording events. So while 
their quantum explanation is not explicitly inconsistent with the first conjunct of (IP), mediating causes could be found only by constructing a new theory. Bell's work has clearly delineated the obstacles that would have to be overcome on that path.

\section{References}

[1] Bell, J.S. [1964]: On the Einstein-Podolsky-Rosen paradox. Physics 1, 195200.

[2] Bell, J.S. [1975]: The theory of local beables. TH-2053-CERN, July 28.

[3] Bell, J.S. [1981]: Bertlmann's socks and the nature of reality. Journal de Physique, Colloque 2, suppl. au numero 3, Tome 42, C2 41-61.

[4] Bell, J.S. [1985]: The theory of local beables. Dialectica 39, 86-96.

[5] Bell, J.S. [1990]: La nouvelle cuisine. In Sarlemijn and Krose, eds., Between Science and Technology, 97-115.

[6] Bell, J.S. [2004]: Speakable and Unspeakable in Quantum Mechanics. Second Revised Edition. Cambridge: Cambridge University Press.

[7] Eberhard, P. [1978]: Bell's theorem and the different concepts of locality. Nuovo Cimento, B46, 392-419.

[8] Einstein, A. [1948]: Quanten-mechanik und Wirklichkeit. Dialectica 2, 32023.

[9] Einstein, A., Podolsky, B., and Rosen, N. [1935]: Can quantum-mechanical description of physical reality be considered complete. Physical Review 47, 777-80.

[10] Healey, R. [2012]: Quantum theory: a pragmatist approach. British Journal for the Philosophy of Science 63, 729-71.

[11] Ismael, J. [2008]: Raid! Dissolving the big, bad bug. Nous 42, 292-307.

[12] Maudlin, T. [2007]: The Metaphysics within Physics. Oxford: Oxford University Press.

[13] Maudlin, T. [2011]: Three roads to objective probability. In Beisbart, C. and Hartmann, S. eds. [2011]. Probabilities in Physics. Oxford: Oxford University Press, 293-319.

[14] Norsen, T. [2011]: John S. Bell's Concept of Local Causality. American Journal of Physics 79, 1261-75.

[15] Price, H. [2012]: Causation, Chance and the rational significance of supernatural evidence. Philosophical Review 121, 483-538. 
[16] Seevinck, M.P., Uffink, J. [2011]: Not throwing out the baby with the bathwater: Bell's condition of local causality mathematically 'sharp and clean", in D. Dieks et al. Explanation, Prediction and Confirmation. Berlin: Springer, 425-50.

[17] Woodward, J. [2003]: Making Things Happen. Oxford: Oxford University Press. 


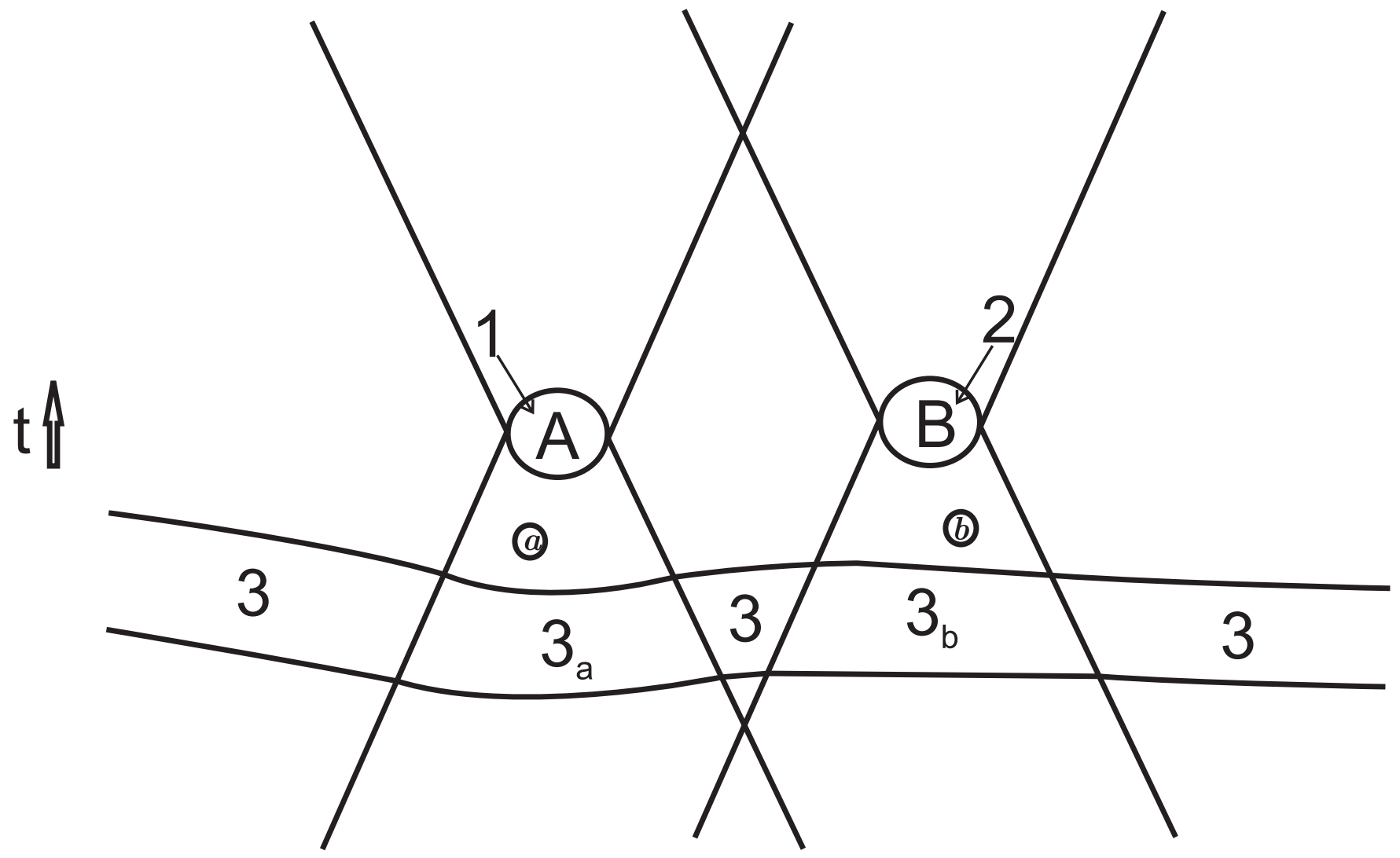

Figure 1: Space-time diagram for LOCAL CAUSALITY 


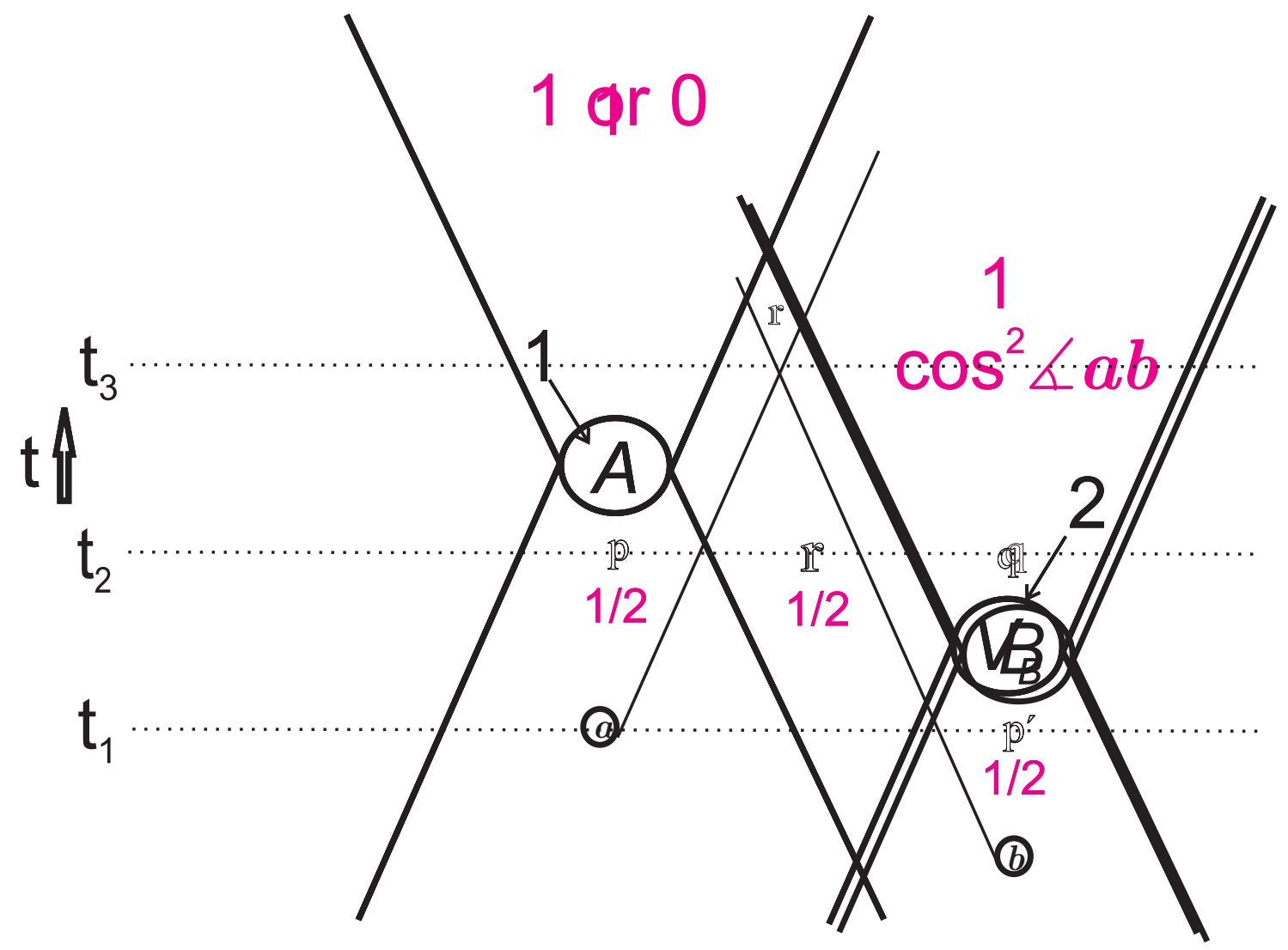

Figure 4: Explaining Bell's correlations

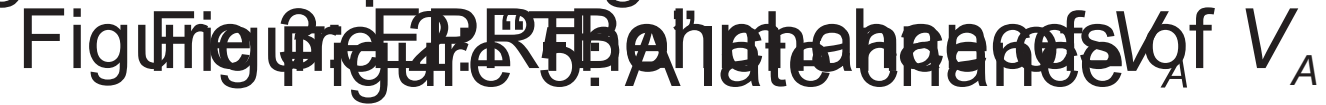

